\title{
Enseigner l'histoire pour éduquer les élèves aux droits humains? Le cas de l'histoire de l'apartheid dans des classes québécoises
}

\author{
Teaching history to educate students about human rights? \\ Teaching the history of apartheid in Quebec classrooms \\ ¿Enseñar la historia para educar a los alumnos a los derechos \\ humanos? El caso de la historia del apartheid en las clases \\ quebequenses
}

\section{Sabrina Moisan}

Volume 47, numéro 2, automne 2019

Éduquer aux sciences humaines et sociales

URI : https://id.erudit.org/iderudit/1066450ar

DOI : https://doi.org/10.7202/1066450ar

\section{Aller au sommaire du numéro}

\section{Éditeur(s)}

Association canadienne d'éducation de langue française

\section{ISSN}

1916-8659 (numérique)

\section{Découvrir la revue}

\section{Citer cet article}

Moisan, S. (2019). Enseigner l'histoire pour éduquer les élèves aux droits humains? Le cas de l'histoire de l'apartheid dans des classes québécoises. Éducation et francophonie, 47(2), 102-122. https://doi.org/10.7202/1066450ar

\section{Résumé de l'article}

Comment l'histoire scolaire contribue-t-elle à une éducation citoyenne portant sur l'enjeu des droits humains? Ce lien entre histoire scolaire et formation citoyenne n'est pas aisé à implanter, et sa raison d'être se voit même parfois contestée. Cet article, qui cherche à contribuer à la réflexion et au débat, s'intéresse aux pratiques d'enseignement mises en place par trois enseignants d'histoire du secondaire (une enseignante et deux enseignants) au sujet de l'histoire de l'apartheid. Dans un premier temps, l'analyse compare les finalités prescrites dans le programme d'études à celles poursuivies par l'enseignante et les deux enseignants. Ensuite, les pratiques mises en place sont analysées de manière à comprendre comment un sujet, comme les luttes antiapartheid, est enseigné et quel peut être son apport pour l'éducation aux droits humains. Les résultats montrent que le programme d'histoire et ses documents afférents (notamment la Progression des apprentissages) ont un effet important sur l'organisation des contenus, et donc de la classe, ce qui n'encouragerait pas la formation citoyenne critique ni une compréhension politique des enjeux des droits humains. L'article contribue ainsi à la réflexion sur les liens entre l'enseignement de l'histoire, l'éducation aux droits humains et l'éducation à la citoyenneté, tout en éclairant également les habitudes professionnelles d'enseignantes et d'enseignants d'histoire au secondaire.

Tous droits réservés @ Association canadienne d'éducation de langue française, Ce document est protégé par la loi sur le droit d’auteur. L’utilisation des 2019 services d'Érudit (y compris la reproduction) est assujettie à sa politique d'utilisation que vous pouvez consulter en ligne. 


\section{Enseigner l'histoire pour éduquer les élèves aux droits humains? Le cas de l'histoire de l'apartheid dans des classes québécoises}

\section{Sabrina MOISAN}

Université de Sherbrooke, Québec, Canada

\section{RÉSUMÉ}

Comment l'histoire scolaire contribue-t-elle à une éducation citoyenne portant sur l'enjeu des droits humains? Ce lien entre histoire scolaire et formation citoyenne n'est pas aisé à implanter, et sa raison d'être se voit même parfois contestée. Cet article, qui cherche à contribuer à la réflexion et au débat, s'intéresse aux pratiques d'enseignement mises en place par trois enseignants d'histoire du secondaire (une enseignante et deux enseignants) au sujet de l'histoire de l'apartheid. Dans un premier temps, l'analyse compare les finalités prescrites dans le programme d'études à celles poursuivies par l'enseignante et les deux enseignants. Ensuite, les pratiques mises en place sont analysées de manière à comprendre comment un sujet, comme les luttes antiapartheid, est enseigné et quel peut être son apport pour l'éducation aux droits humains. Les résultats montrent que le programme d'histoire et ses documents afférents (notamment la Progression des apprentissages) ont un effet important sur l'organisation des contenus, et donc de la classe, ce qui n'encouragerait pas la formation citoyenne critique ni une compréhension politique des enjeux des droits humains. L'article contribue ainsi à la réflexion sur les liens entre l'enseignement 
de l'histoire, l'éducation aux droits humains et l'éducation à la citoyenneté, tout en éclairant également les habitudes professionnelles d'enseignantes et d'enseignants d'histoire au secondaire.

\section{ABSTRACT}

\section{Teaching history to educate students about human rights? Teaching the history of apartheid in Quebec classrooms}

Sabrina MOISAN, University of Sherbrooke, Quebec, Canada

How does history class contribute to citizenship education on the issue of human rights? The connection between history class and citizenship education is not easy to implement, and its reason for being is even disputed at times. This article, which seeks to contribute to the reflection and debate, focuses on the teaching practices of three high school history teachers who teach the history of apartheid. The analysis first compares the objectives set in the program of study to those pursued by the three teachers. Then their practices are analyzed in order to understand how a subject, such as the fight against apartheid, is taught and what its contribution to human rights education can be. The results show that the history program and its related documents (notably the Progression of Learning) have a significant impact on the organization of content, and therefore of the class, which would not encourage the training of critical citizens or a political understanding of human rights issues. The article thus contributes to reflections on the connections between teaching history, human rights and citizenship education, while also looking at the professional practices of high school history teachers.

\section{RESUMEN}

\section{¿Enseñar la historia para educar a los alumnos a los derechos humanos? El caso de la historia del apartheid en las clases quebequenses}

Sabrina MOISAN, Universidad de Sherbrooke, Quebec, Canadá

¿Cómo la historia escolar contribuye a una educación ciudadana centrada en los retos de los derechos humanos? La relación entre historia escolar y formación ciudadana no es fácil de establecer y su propósito a veces se ve pugnado. Este artículo, que trata de contribuir a la reflexión y al debate, se interesa a las prácticas educativas aplicadas por tres maestros de historia de secundaria (una maestra y dos maestros) sobre la historia del apartheid. En un primer momento, el análisis compara las finalidades prescritas por el programa de estudios con la perseguidas por la maestra y los dos maestros. Después, las prácticas utilizadas se analizan con el fin de comprender 
cómo el sujeto, cómo las luchas anti-apartheid, se enseña y cuál puede ser su contribución para la educación a los derechos humanos. Los resultados muestran que el programa de historia y sus documentes relacionados (principalmente Progresión de los aprendizajes) tienen un efecto importante sobre la organización de los contenidos, y por lo tanto sobre la clase, lo que no estimula la formación ciudadana crítica ni la comprensión política de los retos de los derechos humanos. El artículo contribuye pues a la reflexión sobre las relaciones entre enseñanza de la historia, educación a los derechos humanos y educación a la ciudadanía, esclareciendo asimismo los hábitos profesionales de maestros y maestras de historia de secundaria.

\section{INTRODUCTION}

Étudier l'histoire, c'est tenter de saisir toujours mieux la nature humaine. Bloch (1949) disait aussi que s'exercer à mieux connaitre le présent aide à comprendre le passé. Les deux temporalités sont inséparables et devraient ainsi se faire écho dans la classe d'histoire. Avoir prise sur le présent grâce à une perspective historique critique n'est-elle pas l'une des fonctions citoyennes de l'histoire scolaire? Quel intérêt y a-t-il à enseigner l'histoire si celle-ci ne permet pas à l'individu de mieux vivre en société et d'assurer la dignité humaine de tous, sans distinctions sociales, culturelles, de genre, etc.?

Pourtant, les finalités citoyennes en classe d'histoire ne font pas l'unanimité, comme l'a montré le douloureux débat ayant animé la société québécoise de 2006 jusqu'à 2014, sinon jusqu'à nos jours (voir, entre autres, Bouvier, 2007; Dagenais et Laville, 2007; Éthier, Cardin et Lefrançois, 2013). C'est que la citoyenneté est perçue par les uns comme étant suspecte, au service d'un ordre social que l'on ne veut pas: qu'il soit nationaliste, fédéraliste, de gauche ou de droite. Pour les autres, la citoyenneté à l'école est gage d'émancipation de l'élève, libéré des catégories déjà prêtes à penser.

Ce texte s'intéresse à l'une des composantes de la formation citoyenne du $21^{\mathrm{e}}$ siècle et au cœur de laquelle siège la question de la dignité humaine. En effet, l'éducation aux droits humains est un champ florissant dans lequel praticiens, politiques et chercheurs se rencontrent. Le potentiel qu'offre la classe d'histoire pour l'éducation aux droits ou l'inverse n'est toutefois pas encore très clair, malgré des contributions importantes à cet égard (par exemple, Van der Leeuw-Roord, 2014; Vesterdal, 2014).

Mais sur ce sujet aussi règne la controverse. Plusieurs auteurs affirment le potentiel de la classe d'histoire pour ce type de formation, car elle permettrait de mieux comprendre la complexité des enjeux et les rapports de pouvoirs, au passé et au présent (Van der Leeuw-Roord, 2014; Vesterdal, 2014; Wogenstein, 2015). Cette pertinence est 
toutefois remise en question par d'autres chercheurs qui rappellent que cette discipline scolaire est trop souvent associée à la transmission d'une mémoire collective nationale et, sur le plan pédagogique, à la mémorisation, deux caractéristiques ayant peu de portée critique et dépourvues de l'universalisme prôné par l'éducation aux droits humains (Eckmann, 2010; Mihr, 2015).

Cet article propose ainsi une contribution à cette réflexion en s'arrêtant sur le cas québécois afin de mieux comprendre l'apport de l'histoire scolaire à une éducation critique aux droits humains critique. Plus spécifiquement, ce sont les objectifs et les pratiques d'enseignement mises en place à propos de l'histoire de l'apartheid sud-africain qui sont analysés. Le chapitre dans lequel ce thème est enseigné fait partie du programme Histoire et éducation à la citoyenneté (HEC) du $1^{\mathrm{er}}$ cycle du secondaire (Ministère de l'Éducation, du Loisir et du Sport [MELS], 2004). Les données utilisées proviennent d'un projet de recherche ${ }^{1}$ (2013-2017) portant sur l'enseignement de l'histoire de mouvements sociaux pour l'obtention des libertés et des droits civils. L'objectif principal vise à documenter pourquoi et comment les enseignantes et les enseignants d'histoire traitent, en classe l'histoire, de sujets difficiles tels l'apartheid, en lien avec une éducation aux droits humains.

\section{LE CONTEXTE CURRICULAIRE}

Le programme Histoire et éducation à la citoyenneté propose un chapitre sur «la reconnaissance des droits civils et des libertés» dans lequel les enseignantes et les enseignants sont invités à aborder l'un des thèmes suivants: antiracisme (comprenant le mouvement de lutte pour les droits civils des Noirs aux États-Unis et les luttes contre l'apartheid en Afrique du Sud), féminisme, décolonisation ou, de manière comparative et optionnelle, l'Holocauste. Basé sur une approche pédagogique par compétences, le programme suggère ainsi que les enseignantes et les enseignants amènent les élèves de 14 ans à se pencher sur la question des privations et des luttes pour la conquête des libertés et des droits civils actuels, de même que sur celle de la reconnaissance de ces libertés et de ces droits dans le passé (MELS, 2004, p. 364, compétence disciplinaire 1). Les élèves sont également tenus d'interpréter les «retombées de la conquête des libertés et des droits civils pour les collectivités» (MELS, 2004, p. 364, compétence disciplinaire 2) et de réfléchir aux «responsabilités de l'individu dans la conquête et la reconnaissance des libertés et des droits civils» (MELS, 2004, p. 364, compétence disciplinaire 3). Le savoir doit être organisé selon les concepts centraux suivants: liberté, censure, démocratisation, discrimination, dissidence, droits, égalité, répression et ségrégation (MELS, 2004).

1. Projet «Le changement social en contexte démocratique: représentations et pratiques d'enseignantes et d'enseignants", financé par le Fonds de recherche du Québec - Société et Culture (FRQSC), programme Établissement de nouveaux professeurs-chercheurs. 
Les deux premières compétences visent le développement de la perspective historique par l'établissement de liens entre le passé et le présent, de même que le développement de la méthode historique et de l'interprétation. Ces compétences reprennent ainsi des composantes essentielles au développement de la pensée historienne (Seixas et Morton, 2013; Wansink, Akkerman, Zuiker et Wubbels, 2018). La troisième compétence concerne l'éducation à la citoyenneté et vise à ce que les élèves réfléchissent aux valeurs et aux principes ayant guidé les acteurs du passé tout en comparant ceux qui marquent encore la société contemporaine (MELS, 2004).

Le programme est ambitieux, mais fournit peu de détails sur la façon d'enseigner ces sujets. De la même manière, alors que la question des droits humains est centrale, le document présentant la progression des apprentissages pour ce chapitre porte principalement sur la connaissance factuelle des droits (MELS, 2010). Pour sa part, la thématique de l'antiracisme comprend deux sous-thèmes: les luttes des Noirs aux États-Unis et celles en Afrique du Sud. Dans le document du ministère qui précise les connaissances à enseigner, les deux phénomènes sont présentés ensemble.

\section{Tableau 1. Extrait de la Progression des apprentissages}

\subsection{Lutte contre le racisme}

a. Énoncer des droits inscrits dans la Déclaration universelle des droits de I'homme sur lesquels s'appuient les mouvements qui luttent contre le racisme institué (ex.: principe selon lequel tous les êtres humains naissent libres et égaux en dignité et en droits)

b. Indiquer des préjugés à l'égard des Noirs aux États-Unis dans la première moitié du $20^{\text {e }}$ siècle et en Afrique du Sud avant l'abolition de l'apartheid (ex.: discrimination, ségrégation, absence de droits civils)

c. Nommer des acteurs associés à la lutte contre la ségrégation raciale (ex.: Rosa Parks, Martin Luther King, Nelson Mandela)

d. Indiquer des actions entreprises par des citoyens ou des États pour lutter contre le racisme institué au cours du $20^{\mathrm{e}}$ siècle (ex.: résistance passive, manifestation, émeutes)

e. Indiquer des réactions face au mouvement pour la reconnaissance des libertés et des droits civils (ex.: répression, emprisonnement)

f. Indiquer des gains obtenus par les Noirs, notamment aux États-Unis et en Afrique du Sud (ex.: Civils Rights Act, Voting Rights Act, abolition de l'apartheid)

Source: MELS, 2010, p. 29.

Les luttes pour les droits civils des Noirs aux États-Unis ainsi que celles menées contre l'apartheid en Afrique du Sud sont amalgamées et présentées de manière factuelle (objective) sans tenir compte du contexte historique spécifique à chacune. Or, la comparaison en histoire perd de sa pertinence si les contextes propres à chaque événement ou à chaque phénomène ne sont pas pris en compte (Boix-Mansilla, 2000). Tel qu'il est présenté, ce document (la Progression des apprentissages) n'encourage pas les enseignantes et les enseignants à considérer les contextes. 


\section{ÉDUCATION AUX DROITS HUMAINS DANS LA CLASSE D'HISTOIRE}

Depuis les catastrophes humaines causées par les deux guerres mondiales, la question de l'éducation aux droits humains est une préoccupation partagée par les membres de la communauté internationale, mais dont les engagements à cet égard varient d'un pays à l'autre, voire d'une province à l'autre. L'Organisation des Nations Unies recommande que l'éducation aux droits humains se fasse sur trois plans: SUR, PAR et POUR les droits humains. Enseigner SUR concerne en grande partie l'acquisition de connaissances sur les droits humains, dont le contenu de la Déclaration universelle des droits de l'homme; enseigner $P A R$ vise l'application des droits humains en classe (p. ex., les règles de la classe); et enseigner $P O U R$ les droits humains poursuit le but de responsabiliser les élèves dans la lutte pour le respect de ces droits (Déclaration des Nations Unies sur l'éducation et la formation aux droits de l'homme, article 2, paragraphe 2, 2011).

Afin d'approfondir ces prescriptions internationales, Tibbitts (2002) propose un modèle théorique prenant la forme d'une pyramide d'apprentissage à trois niveaux: la sensibilisation, la responsabilisation et la transformation. Au premier niveau de la sensibilisation, les élèves sont initiés aux droits humains à leur portée universelle et aux cas de violation de ces droits ici ou ailleurs. Ce sont alors les dimensions morale et philosophique, centrées sur la notion d'humanité, de l'éducation aux droits de l'homme, qui prédominent (Barton, s. d.). Le deuxième niveau, soit la responsabilisation, vise à amener les élèves à réfléchir à la question de la sauvegarde et de la protection des droits humains en favorisant une meilleure compréhension du rôle des institutions locales, nationales et internationales dans des situations spécifiques. Il s'agit d'un apprentissage plus politique menant à une compréhension plus systémique d'un enjeu de droits humains, mais qui relève aussi de la morale en faisant réfléchir les élèves aux responsabilités et au pouvoir d'action tant des individus que des institutions. Au troisième niveau, à savoir le modèle transformationnel, les élèves deviennent des acteurs informés, avertis et sensibilisés, capables d'agir conformément aux principes des institutions chargées de la protection des droits humains (Tibbitts, 2002). Comment l'histoire des luttes contre l'apartheid enseignée au Québec s'inscrit-elle dans ce modèle théorique?

Pour l'école québécoise, la Progression des apprentissages (voir le tableau 1) prescrite par le programme d'histoire montre que la dimension de la sensibilisation aux droits humains est bien présente en demandant aux élèves de nommer des exemples historiques limitant les droits des personnes noires sous le régime d'apartheid. Le niveau de la responsabilisation est également abordé dans la compétence 3, mais ne concerne que le rôle du citoyen dans la lutte pour les droits humains, omettant de mentionner celui des institutions. Ce portrait fait écho aux résultats de den Heyer (2018) voulant que le personnel enseignant ainsi que les élèves aient tendance à attribuer le pouvoir d'action aux individus plutôt qu'aux actions collectives organisées. 
La dimension transformationnelle est, pour sa part, absente des prescriptions, qui n'engagent pas le personnel enseignant à mener les élèves vers l'action et le changement social.

\section{Enseigner l'histoire d'un changement social}

Les séquences d'enseignement sur l'apartheid et les mouvements de luttes visant à l'abolir peuvent prendre différentes formes. Elles pourraient être de nature descriptive, c'est-à-dire qu'y seraient décrits le contexte historique, les raisons menant à l'instauration de ce système, les acteurs impliqués, leur rôle dans ce système ainsi que les conséquences pour chacun. Il est également possible de présenter l'histoire de cette période en focalisant l'enseignement sur son caractère transformateur, au sens de Tibbitts (2005), c'est-à-dire en cherchant à comprendre non seulement le système et ses conséquences, mais aussi les conditions qui ont permis ou empêché l'abolition de l'apartheid. Ce faisant, l'enseignement de l'histoire des luttes contre l'apartheid et pour les droits humains peut être fait de manière à développer les compétences historiennes et critiques des élèves (Osler et Starkey, 2010) en analysant différentes perspectives et en prenant en considération les motivations des différents acteurs en fonction du contexte historique dans lequel ils agissent (Endacott et Brooks, 2013; Wansink et al., 2018). De même, en s'arrêtant sur le présent de ce pays où la ségrégation raciale semble toujours sévir malgré l'abolition de l'apartheid (Borer, 2003; Wassermann, 2017), de même que sur la situation des droits humains dans d'autres sociétés contemporaines, dont la leur, les élèves verraient le sujet non plus comme le fait d'un passé lointain, mais plutôt comme un enjeu encore bien actuel. Ce type de formation historienne répondrait à une éducation plus politique aux droits humains (Barton, s. d.; 2015) tout en permettant aux élèves de mieux comprendre le phénomène du changement social.

Le phénomène du changement social doit être décomposé en différents éléments observables dans l'enseignement comme le contexte historique dans lequel le phénomène prend place; les facteurs explicatifs ou les influences sur le changement; les obstacles au changement; les acteurs pour et contre le changement; l'organisation des actions et des moyens mis en œuvre pour réaliser ou empêcher le changement; le rythme du changement (transformation ou révolution); la relation avec la situation actuelle (Bajoit, 2003; Éthier, Lefrançois, Demers, 2013; Rocher, 1992).

Par ailleurs, l'étude des luttes antiapartheid implique aussi d'aborder un concept aux implications morales importantes, c'est-à-dire le racisme. Ce concept crée de l'inconfort (Zembylas, 2015) et est hautement épineux (Teeger, 2015). Or, des études ont montré que les enseignantes et les enseignants d'histoire ont tendance à refroidir ces sujets pour éviter d'entrer dans les zones désagréables (Wansink et al., 2018). Par exemple, en Afrique du Sud, le personnel enseignant présente l'apartheid en montrant les «deux côtés de l'histoire», c'est-à-dire que des personnes blanches 
étaient contre ce système et que des personnes noires ont aussi commis des actes de violence contre les "Blancs et Blanches", ce qui tend à banaliser les divisions sociales de l'époque et à laisser dans l'ombre la dimension institutionnelle du phénomène (Teeger, 2015).

\section{ÉLÉMENTS MÉTHODOLOGIQUES}

Ce chapitre présente les résultats d'une étude qualitative multicas (2014-2018, financée par le Fonds de recherche du Québec - Société et culture) menée auprès de trois enseignants du secondaire (une enseignante et deux enseignants) et de leurs élèves de 14 ans en moyenne dans le cadre d'un cours d'histoire de deuxième secondaire$^{2}$. Les trois écoles sont situées dans des zones urbaine, semi-urbaine et rurale. L'échantillon accidentel et non représentatif a été constitué grâce aux commissions scolaires, qui ont lancé l'invitation à participer à cette recherche. Les enseignants volontaires ont été acceptés.

Afin de comprendre la façon dont ces enseignants percevaient le concept central du changement social, la théorie et les outils méthodologiques des représentations sociales ont été mobilisés. Les représentations sociales sont définies comme un système de pensées et de théories empruntant à la fois aux connaissances traditionnelles et scientifiques, utilisées par les individus pour donner un sens au monde (Abric, 1994; Garnier, 2015). Les représentations sociales fournissent des lignes directrices pour l'action et la prise de décision. Elles sont donc importantes pour l'éducation, car elles aident à déterminer les objectifs d'apprentissage et à organiser la connaissance scolaire. Il est toutefois difficile de déterminer le degré d'influence des représentations sociales sur l'enseignement, car d'autres aspects de la profession enseignante interviennent dans l'organisation du travail, notamment les contraintes liées à la forme scolaire (répartition du temps, enchaînement des chapitres) et celles émanant des normes institutionnelles (Demers et Éthier, 2013; Lautier, 2006; Moisan et Saussez, 2019). C'est pourquoi les représentations sociales sont analysées ici en lien avec les pratiques enseignantes, y compris les discours des enseignantes et des enseignants sur leur travail.

Pour collecter le discours sur les objectifs éducatifs du personnel enseignant, des entretiens semi-structurés d'une heure en moyenne ont été réalisés avant et après l'enseignement du chapitre dans lequel s'inscrit l'étude de l'apartheid. Les entretiens comprenaient des tâches d'association hiérarchisées continuées (par exemple, comment définissez-vous le concept de «changement social»?) et une liste d'association de neuf éléments parmi lesquels les membres du personnel enseignant devaient choisir trois exemples décrivant le mieux leur compréhension du changement social.

2. Au Québec, l'école secondaire compte 5 niveaux, soit de la $7^{\mathrm{e}}$ à la $11^{\mathrm{e}}$ année. Les élèves ont entre 12 et 17 ans. 
Ensuite, des observations non participantes en classe ont été réalisées, le temps de l'enseignement du chapitre entier (environ 10 heures d'observation par enseignant), afin de comprendre la nature du savoir enseigné sur un phénomène historique (les facteurs explicatifs mis de l'avant, les concepts développés, les perspectives présentées dans l'enseignement du changement social). Un groupe d'élèves (en moyenne, 25 par classe) par enseignant a été suivi, et tout le matériel pédagogique utilisé ainsi que des copies des examens finaux des élèves ont été recueillis. Ces documents ont fait l'objet d'une analyse thématique de contenu visant à mieux comprendre comment chaque sujet historique était présenté. Une analyse de contenu thématique selon Bardin (2007) a été réalisée sur toutes les données collectées (transcriptions d'interviews, notes d'observation et audio, matériel pédagogique, travaux d'élèves) en fonction du cadre d'analyse des pratiques en éducation aux droits humains ainsi qu'à l'aide des catégories définissant le changement social telles que présentées dans le chapitre précédent (Bajoit, 2003; Rocher, 1992). L'analyse de l'ensemble des données a été réalisée par deux codeuses (l'auteure et une assistante) afin d'assurer une meilleure fiabilité des interprétations.

\section{RÉSULTATS}

Les résultats sont présentés de manière verticale, c'est-à-dire qu'un arrêt est fait sur chaque cas et sur chaque enseignant. Les objectifs éducatifs, la séquence d'enseignement et l'évaluation des élèves qui est proposée sont alignés de manière à rendre compte de la cohérence interne de la pratique enseignante.

\section{Enseignante 1}

Cette enseignante travaille dans un milieu rural. Elle a sept ans d'expérience en enseignement de l'histoire. Son objectif éducatif pour l'enseignement de l'apartheid est de permettre aux élèves de réfléchir à la question des droits humains. Elle voit le chapitre sur la reconnaissance des libertés et des droits civils comme étant l'aboutissement logique de l'année scolaire:

L'objectif était de terminer l'année avec les droits et libertés. Puis, ils se rendront compte que, finalement, la Charte [Charte canadienne des droits et libertés] et les droits de la personne sont bons. Et bon, mais si personne ne fait rien, ils n'existeront plus. (Entrevue préobservation)

Pendant un cours typique, les élèves ont vu des présentations PowerPoint, analysé des textes dans leur cahier d'étude et ont eu de longues discussions, en particulier sur certains des droits inscrits dans la Déclaration universelle des droits de l'homme. 
L'enseignante a consacré 2 cours de 75 minutes chacun aux mouvements antiracistes aux États-Unis et en Afrique du Sud. Pour initier les élèves au concept d'apartheid, elle leur a donné des photographies illustrant la ségrégation (restaurants, toilettes, transports, parcs, etc.). L'enseignante a ensuite défini l'apartheid comme étant un système de ségrégation entre personnes blanches et noires. Elle a souligné le fait que le système privilégiait les Blancs, et que les Noirs protestaient contre la discrimination inhérente au système.

L'enseignante a présenté la mort du militant Steve Biko comme un moment charnière dans la lutte contre l'apartheid. Elle a fait jouer la chanson antiapartheid Asimbonanga (Mandela) de Johnny Clegg pour présenter les «deux côtés de l'histoire». Puis, elle a demandé aux élèves:

De quelle couleur est Johnny Clegg? C'est un Blanc. Il y avait des Blancs qui n'étaient pas d'accord avec l'apartheid. John F. Kennedy, le président américain, a serré la main de Martin Luther King. Il était contre l'apartheid, mais des lois aux États-Unis empêchaient toujours les Noirs d'aller à l'école.

Cet extrait du discours de l'enseignante illustre bien le fait que l'histoire des deux mouvements antiracistes est imbriquée dans ses leçons.

\section{Évaluation des élèves}

Comme tâche synthèse, les élèves avaient à mettre en parallèle des exemples de discrimination, de ségrégation et d'oppression à l'égard des personnes noires (d'Afrique du Sud et des États-Unis, ensemble dans la $2^{\mathrm{e}}$ colonne) et des Juifs (dans la $3^{\mathrm{e}}$ colonne).

L'enseignante a évalué la compréhension des élèves à l'aide de la question suivante: "Qui est le plus responsable de la reconnaissance des droits humains: les citoyens ou les gouvernements?» Il s'agissait d'une synthèse des contenus couverts en classe, organisés selon les concepts centraux du cours et les mouvements sociaux étudiés.

Les réponses des élèves démontrent une réflexion assez bien organisée sur la question des mouvements de défense des droits humains, bien que leurs exemples confondent souvent différents événements historiques et ignorent parfois le contexte spatio-temporel. Les élèves se concentrent sur des individus isolés pour expliquer le changement et estiment que c'est surtout le gouvernement qui garantit et protège les droits humains:

En fait, en Afrique et en Amérique, [les Noirs] ont été réduits à l'esclavage. Plus loin dans l'histoire, dans les années 1900, ils étaient complètement séparés des Blancs; différents restaurants, différentes salles de bains, différents quartiers, etc. [...] Cependant, certains [acteurs] se distinguent plus 
que d'autres, comme Martin Luther King, qui a prononcé de nombreux discours aux États-Unis. Il s'est battu contre la ségrégation raciale sans violence. [...] Il y en a beaucoup d'autres comme Nelson Mandela, Gandhi, etc. - Copie d'élève 10

Des gens «ordinaires» comme Gandhi, qui souhaitaient libérer l'Inde de l'empire américain. Ou Martin Luther King, qui a défendu les Noirs en Afrique du Sud contre l'apartheid. Certains, qui défendaient leurs droits, ont été emprisonnés ou tués, mais ils n’ont jamais abandonné. - Copie d'élève 14

Dans l'extrait suivant, l'élève laisse entendre, bien que de manière confuse, que les Africains, de nos jours, ne savent pas qu'ils ont des droits et qu'ils devraient être informés par «le gouvernement», qui est également celui qui offre et protège ces droits:

Si le gouvernement informait les citoyens de leurs droits, les conflits seraient moins nombreux, je crois. [...] Comme en Afrique, qui va leur dire qu'ils ont des droits? Même s'ils le savaient, comment savez-vous qu'ils ne seront pas massacrés comme les Noirs [l'ont été]? - Copie d'élève 22

Il appert ainsi que les élèves ont parfois confondu les deux mouvements antiracistes qu'ils avaient étudiés. De plus, les élèves semblent considérer l'expérience aux ÉtatsUnis et en Afrique du Sud comme si elles étaient identiques, sans prendre en compte les différentes circonstances sociales ou historiques.

Cela pourrait être dû à l'approche de l'enseignante qui, comme cela est prescrit dans le programme et le document Progression des apprentissages, a demandé à ses élèves de comparer systématiquement les situations des personnes noires aux États-Unis et en Afrique du Sud, en insistant sur les similitudes.

Enfin, il semble que l'objectif de l'enseignante, qui était de sensibiliser les élèves à la question de l'importance des droits humains, ait été atteint en grande partie. En effet, l'échantillon de réponses des élèves montre que tous ont affirmé l'importance d'assurer l'accès aux droits humains et de les protéger partout dans le monde.

\section{Enseignant 2}

Cet enseignant travaille dans un environnement semi-urbain et possède 15 ans d'expérience en enseignement de l'histoire et de la géographie. Ses élèves suivent un programme de vocation (santé globale) et, pour cette raison, ils disposent de moins de temps pour ce chapitre (8 cours au lieu de 12 ). Son objectif principal, pour ce chapitre sur la reconnaissance des droits civils et des libertés, est de "couvrir le programme», c'est-à-dire passer au travers des thématiques et des contenus prescrits. Il considère 
néanmoins que ce chapitre est particulièrement intéressant pour les élèves, car il est plus contemporain.

En raison d'un manque de temps et parce qu'il considère que "ce sujet est plus simple», il consacre moins d'une leçon de 75 minutes à l'apartheid. Pendant cette leçon, il a très peu parlé. Il a plutôt proposé aux élèves de répondre à une série de questions à l'aide du manuel scolaire (Hudon et Gervais, 2006). Les élèves devaient trouver des réponses aux questions suivantes: «Qu'est-ce que l'apartheid? Dans quel pays et dans quelles circonstances le régime d'apartheid a-t-il été institué? Qui a bénéficié de l'apartheid? Justifiez votre réponse. Quand l'apartheid a-t-il pris fin? Nommez trois personnes qui se sont battues pour mettre fin à l'apartheid et décrivez leurs rôles.»

\section{Évaluation des élèves}

Pour préparer l'examen final, les élèves ont reçu une feuille d'étude présentant des dates et des faits importants à connaître. Les questions suivantes y sont suggérées: «Qu'est-ce que l'apartheid? Quand était-il en vigueur? Comment ce système s'est-il terminé? Comment les droits des opposants ont-ils été reconnus?»

Lors de l'examen, les élèves ont eu à répondre à la question suivante: "Qu'est-ce que l'apartheid et comment s'est-il terminé?» Les réponses obtenues sont presque identiques à celle du manuel scolaire de référence:

L'apartheid est la séparation des races. Le système a commencé en 1948 en Afrique du Sud et a pris fin en 1996 avec une nouvelle constitution garantissant les droits de tous les citoyens, quelle que soit leur couleur de peau. F. de Klerk et N. Mandela ont mis fin à l'apartheid. - Copie d'élève 3

En général, les élèves semblaient avoir bien réussi. En ce sens, l'enseignant a atteint son objectif de couvrir et de faire apprendre les connaissances factuelles du programme.

\section{Enseignant 3}

Cet enseignant travaille en milieu urbain. Il a 13 ans d'expérience dans l'enseignement de l'histoire au secondaire. Il espère qu'avec ce chapitre, ses élèves comprendront l'idée que la lutte pour l'égalité subsiste aujourd'hui et qu'il existe différentes stratégies, pacifiques ou violentes, pour mener ces luttes. Il a consacré 3 cours de 75 minutes à l'apartheid. 
L'introduction du cahier d'apprentissage qu'il a construit pour ses élèves met côte à côte les mouvements américain et sud-africain, comme on les retrouve dans les documents officiels. Son enseignement est magistro-centré et dialogué.

Il a présenté l'apartheid comme étant le résultat d'une loi promulguée par un gouvernement blanc en 1948 et mettant en œuvre la ségrégation et la discrimination à l'égard des "personnes non blanches». Il souligne ensuite que la résistance à cette loi s'est rapidement organisée afin de mettre fin à l'oppression de l'État. Il considère la répression du soulèvement de Soweto, en 1976, comme un moment charnière de la lutte. Il affirme aux élèves :

Lorsque les Noirs ont manifesté à Soweto, en 1976, la police a commencé à tirer sur les manifestants. À cause de cet événement, le soulèvement a attiré l'attention de l'opinion publique mondiale, et la planète entière a pris conscience de ce qui se passait en Afrique du Sud.

Il s'est ensuite concentré sur le rôle de Nelson Mandela et du Congrès national africain en tant qu'acteurs derrière les manifestations pacifiques, puis armées. Il a mentionné que Mandela avait été arrêté et condamné à la réclusion à perpétuité. Il a souligné que Mandela avait le soutien d'organisations internationales qui critiquaient l'apartheid, boycottaient les produits d'Afrique du Sud et refusaient d'autoriser les athlètes sud-africains à participer aux Jeux olympiques. Il a présenté le rôle du gouvernement canadien en insistant sur les liens entre l'économie et la politique internationale de la manière suivante:

Au Canada, [le premier ministre] Mulroney sera un fervent partisan de l'abolition de l'apartheid et promulguera des lois afin que les entreprises canadiennes cessent de faire affaire avec des entreprises sud-africaines. Nous voulions faire pression sur eux. Si les entreprises sud-africaines ne gagnent pas d'argent, elles feront pression sur leur gouvernement pour qu'il modifie les lois afin de gagner de l'argent.

Il a poursuivi en affirmant que cette pression avait finalement contraint le gouvernement sud-africain à abolir l'apartheid en 1991, à libérer Mandela et à organiser les premières élections multiraciales en 1994.

Lors de son entretien, l'enseignant a souligné que Mandela utilisait des moyens pacifiques comme Luther King, mais qu'il a aussi soutenu plus tard la violence, comme Malcolm X. Il a déclaré qu'il est important de montrer les actes de violence commis par Mandela, car celui-ci est souvent présenté comme un champion de la non-violence. Il souhaitait également que ses élèves se demandent si la violence est nécessaire dans la lutte pour les droits. Il leur a demandé: 
Le mouvement féministe, la décolonisation ont souvent été violents. La violence est-elle nécessaire pour réaliser les droits? Ce sont plus ou moins les types de questions que je veux leur poser.

\section{Évaluation des élèves}

Pour ce chapitre, l'enseignant a conçu une épreuve combinant des questions de connaissances factuelles et l'analyse de documents en vue de répondre à des questions courtes et à une question ouverte (qui ne porte pas sur l'Afrique du Sud). La première question de l'examen est une série de déclarations que les élèves doivent situer soit en Afrique du Sud, soit aux États-Unis. Deux des six déclarations concernent l'Afrique du Sud: «les premières élections multiraciales ont eu lieu en 1994 (réponse fournie dans le document); les lois divisaient la population en catégories: Blancs, Noirs, "Métis" et autres".

Dans l'autre question sur l'Afrique du Sud, les élèves doivent comparer les actions de Nelson Mandela à celles de Martin Luther King et de Malcolm X. Les documents du dossier présentent une courte biographie de chacun. Voici un exemple représentatif des réponses des élèves en ce qui concerne Malcolm X et N. Mandela:

Tous deux se sont battus pour leurs droits, ils ont commis des actes assez violents et ont affirmé que la non-violence n'était plus efficace. - Copie d'élève 15

Si cet enseignant souhaitait d'abord que ses élèves réfléchissent aux luttes encore à faire, il a peu abordé celles-ci en classe. Les réponses données par les élèves à ce sujet ne dépassent guère la comparaison des faits présentés dans les documents offerts dans l'examen.

\section{DISCUSSION}

D’emblée, les résultats de cette étude montrent que les pratiques enseignantes s'inspirent, en partie, des prescriptions du programme d'étude. En effet, les contenus enseignés sont organisés comme dans le document de la Progression des apprentissages (MELS, 2010), qui met en parallèle les luttes pour les droits civils des Noirs aux États-Unis et celles menées contre l'apartheid. Par ailleurs, le programme propose aussi une approche par compétences disciplinaires, qui semble avoir très peu influencé les pratiques enseignantes. Ce premier constat illustre l'importance prioritaire qu'accordent les enseignantes et les enseignants aux contenus historiques plutôt qu'au développement des compétences des élèves, ce qui est corroboré par d'autres études (Boutonnet, 2013; Demers, 2011; Moisan et Saussez, 2019). 
Toutefois, le programme propose aux enseignantes et aux enseignants de n'aborder que l'un des thèmes suggérés, alors que l'enseignante et les deux enseignants ont choisi de les traiter tous. Cette décision a eu pour effet de réduire le temps imparti à chacune des thématiques, qui ont été survolées et insérées dans une sorte de matrice générant un récit canonique des luttes pour la liberté et les droits.

En conséquence, l'enseignante et les deux enseignants ont organisé leurs cours sur l'antiracisme en mettant en parallèle les similitudes et, dans une moindre mesure, les différences entre les luttes antiapartheid (1948-1994) et celles menées aux ÉtatsUnis dans les années 1960. Ce faisant, le contexte historique dans lequel s'insère l'apartheid a été escamoté, empêchant ainsi une réflexion sur la durée et l'ampleur de son incidence sur la société. Le fonctionnement de l'apartheid au quotidien et sa signification pour les différents acteurs sociaux sont éludés. L'expérience des victimes et leur agentivité ne sont pas présentées. Il n'y a aucune analyse de sources ou de témoignages ni en classe ni dans les outils didactiques. L'oppression du système est présentée comme étant une réponse à des manifestations, et non comme la violence constante exercée par l'État contre un segment de la population. Cela empêche de comprendre la discrimination structurelle et ses effets à long terme (Teeger, 2015).

Pour expliquer le changement social, l'enseignante et les deux enseignants ont focalisé leur enseignement sur deux acteurs: Nelson Madela et Frederick de Klerk. Mandela est de loin l'acteur central, mais son rôle est souvent limité par le fait qu'il a résisté à l'apartheid, puis a négocié les termes de son démantèlement. Les circonstances dans lesquelles il a agi ne sont pas explicites. Le président de Klerk est présenté comme la personne qui a mis fin à l'apartheid sous la pression internationale. Plusieurs acteurs impliqués sont ainsi laissés dans l'ombre: femmes, travailleurs du mouvement de résistance, alliés Blancs, Métis et Indiens, avocats, communistes. Les acteurs qui se sont positionnés contre le changement ne sont pas nommés. L'opposant est le gouvernement nationaliste, incarné par la police et l'oppression. Ainsi, les personnes blanches ne sont pas apparues comme des actrices, et les privilèges qu'elles tirent du système ne sont pas non plus pris en compte.

Les cours observés se sont concentrés sur la façon dont la population noire a lutté contre le système d'oppression, par les manifestations, les grèves, le boycottage, la publication de brochures et de livres, les émeutes et la guérilla. Ces actions sont toutefois présentées sans le contexte de leur organisation, ce qui ne permettrait pas de comprendre la complexité du changement social (Bajoit, 2003).

Les prescriptions du programme d'études induisent une présentation simpliste du phénomène. On parle de racisme sans parler de racialisation, ce qui empêche de saisir que les rôles et les comportements attribués aux personnes noires et blanches sont le résultat d'une construction sociale, qui peut elle-même être un objet d'étude, d'autant plus que le racisme systémique est un enjeu très actuel dans la société québécoise et canadienne. Les enseignants auraient pu amener les élèves à comprendre 
ce que signifie cette expression pour les gens qui en sont victimes et pour ceux qui en bénéficient.

Ces résultats sont néanmoins semblables à ceux obtenus auprès d'enseignantes et d'enseignants d'histoire en Afrique du Sud (Teeger, 2015) et aux États-Unis (Swalwell, Pellegrino et View, 2015; Washington et Humphries, 2011), où ces derniers évitent de s'arrêter sur le problème du racisme. Dans ces sociétés, cependant, le personnel enseignant est confronté à des élèves appartenant aux groupes dont il est question dans le cadre du cours. Ce n'est pas le cas au Québec, où les élèves ne sont pas directement touchés. Est-il possible que les enseignantes et les enseignants ressentent néanmoins le besoin d'être les pacificateurs des relations intergroupes? Il est vrai que le Québec n'est pas exempt de racisme, loin de là, mais cette hypothèse appelle à des recherches plus poussées, notamment sur les savoirs et les représentations sociales du personnel enseignant à l'égard du racisme.

Bien que la question des droits humains occupe une place centrale dans le chapitre à l'étude, la nature de l'éducation aux droits de l'homme proposée par le programme et ses documents afférents mène le personnel enseignant à privilégier les savoirs factuels sur les droits ainsi que la dimension de la sensibilisation à l'importance de ceux-ci pour l'humanité. De plus, l'enseignement des droits humains est centré sur l'apprentissage du contenu conceptuel (oppression, discrimination, répression). Pour ce faire, les enseignantes et les enseignants parlent des droits humains et présentent des exemples historiques de violations et de luttes en consacrant très peu de temps au contexte historique, aux enjeux systémiques plus larges et à la complexité de l'organisation de la résistance et des revendications.

Les dimensions de la responsabilité et de la transformation de la société actuelle qui sont associées à l'éducation aux droits humains sont à peine effleurées, à la fois dans les documents officiels et les pratiques d'enseignement observées. Ainsi, bien que deux compétences disciplinaires sur trois invitent les enseignantes et les enseignants à faire des liens entre le passé et le présent, ceux-ci sont absents des leçons observées.

\section{CONCLUSION}

Cette étude a montré l'importance que prennent les contenus historiques prescrits par les documents officiels dans l'organisation de l'enseignement de l'histoire au secondaire. Les contenus semblent en effet avoir priorité sur l'approche pédagogique par compétences qui y est préconisée. Ainsi, plutôt que de promouvoir des compétences critiques propres à une éducation aux droits humains et à la citoyenneté, l'enseignement observé reste descriptif et schématique. L'enseignante et les deux enseignants de cette enquête ont néanmoins atteint leur objectif, qui était de sensibiliser leurs élèves à l'importance des droits humains. 
L'approche conceptuelle préconisée par le programme d'études autour de la ségrégation, de l'oppression et de la discrimination est peut-être pertinente, mais elle n'a pas été mobilisée comme grille d'analyse pour comprendre l'apartheid et les résistances qu'il a générées. Les concepts de colonisation, d'impérialisme ou de domination n’ont pas été mobilisés, alors qu'ils ont théoriquement été développés dans les chapitres précédents du programme d'histoire, dont celui sur la colonisation de l'Afrique lors de la période de l'industrialisation. Puisque les élèves ont vu l'effet de la colonisation, l'enseignante et les deux enseignants auraient pu tabler sur cela pour montrer comment les luttes pour la décolonisation ont également marqué les mouvements de revendications en Afrique du Sud (Mandela, 2010).

Le fait de ne pas aborder le phénomène de racialisation et des injustices qu'il entraîne priverait les élèves d'un outil de compréhension du passé et du présent (Teeger, 2015). Cette situation s'explique-t-elle par le malaise des enseignantes et des enseignants québécois d'histoire à aborder le problème du racisme systémique passé et présent au Canada ou s'explique-t-elle simplement par le fait que le programme n'y convie pas? Certes, ces objectifs prennent du temps et requièrent une maîtrise des faits historiques et des dynamiques animant le fonctionnement social et politique. Chose certaine, la représentation de l'enseignement de l'histoire comme étant le lieu de la transmission d'une culture historique centrée sur l'acquisition de faits semble encore prédominante. Ces résultats interpellent la nature des programmes d'histoire et peut-être aussi la formation des maîtres, qui semblent favoriser assez peu un enseignement de l'histoire qui aurait une portée citoyenne, critique et transformative.

\section{Références bibliographiques}

ABRIC,J.-C. (1994).L'organisationinterne des représentations sociales: système central et système périphérique. Dans C. Guimelli (dir.), Structures et transformations des représentations sociales (p. 73-84). Neuchâtel, France: Delachaux et Niestlé.

BAJOIT, G. (2003). Le changement social.Approche sociologique des sociétés occidentales contemporaines. Paris, France: Armand Colin.

BARDIN, L. (2007). L'analyse de contenu. Paris, France: Presses universitaires de France.

BARTON, K. C. (s. d.) Human rights. Document inédit. 
BARTON, K. C. (2015). Young adolescents' positioning of human rights: Findings from Colombia, Northern Ireland, Republic of Ireland and the United States. Research in Comparative and International Education, 10(1), 48-70.

BLOCH, M. (1949). Apologie pour l'histoire ou le métier d'historien. Paris, France: Armand Colin.

BOIX-MANSILLA, V. (2000). Historical understanding: Beyond the past and into the present. Dans P. N. Stearns, P. Seixas et S. Wineburg (dir.), Knowing, teaching \& learning history. National and international perspectives (p. 390-417). New York, NY: New York University Press.

BORER, T. A. (2003). A taxonomy of victims and perpetrators: Human rights and reconciliation in South Africa. Human Rights Quarterly, 25(4), 1088-1116.

BOUTONNET, V. (2013). Les ressources didactiques: typologie d'usages en lien avec la méthode historique et l'intervention éducative d'enseignants d'histoire au secondaire (Thèse de doctorat, Université de Montréal). Repéré à https:// papyrus.bib.umontreal.ca/xmlui/bitstream/handle/1866/10105/Boutonnet_Vincent_ These_2013.pdf?sequence $=6$ \&isAllowed $=y$

BOUVIER, F. (2007). Quand l'histoire nationale devient problématique au nom de l'éducation à la citoyenneté: phénomène à inverser. Bulletin d'histoire politique, 15(2), 89-106.

DAGENAIS, M. et LAVILLE, C. (2007). Le naufrage du projet de programme d'histoire «nationale». Retour sur une occasion manquée accompagné de considérations sur l'éducation historique. Revue d'histoire de l'Amérique française, 60(4), 517-550.

DEMERS, S. (2011). Relations entre le cadre normatif et les dimensions téléologique, épistémologique, et praxéologique des pratiques d'enseignants d'histoire et éducation à la citoyenneté: étude multicas (Thèse de doctorat inédite, Université du Québec à Montréal). Repéré à https://archipel.uqam.ca/5079/1/D2349.pdf

DEMERS, S. et ÉTHIER, M.-A. (2013). Rapprochement entre curriculum, savoirs savants et pratiques enseignantes en enseignement de l'histoire: l'influence de l'épistémologie pratique, Éducation et didactique, 7-2. Repéré à http:// educationdidactique.revues.org/1743

DEN HEYER, K. (2018). Historical agency: Stories of choice, action, and social change. Dans S. A. Metzer et L. McArthur Harris (dir.), The Wiley international handbook of history teaching and learning (p. 227-252). Medford, MA: Wiley Blackwell. 
ECKMANN, M. (2010). Exploring the relevance of Holocaust education for human rights education. Prospects, 40(1), 7-16.

ENDACOTT, J. et BROOKS, S. (2013). An updated theoretical and practical model for promoting historical empathy. Social Studies Research and Practice, 8(1), 41-58.

ÉTHIER, M.-A., CARDIN, J.-F. et Lefrançois, D. (2013). Cris et chuchotements: la citoyenneté au cœur de l'enseignement de l'histoire au Québec. Revue d'histoire de l'éducation, 25(2), 87-107.

ÉTHIER, M.-A., LEFRANÇOIS, D. et DEMERS, S. (2013). An analysis of historical agency in Québec history textbooks. Education, Citizenship and Social Justice, 8(2), 119-133.

GARNIER, C. (2015). Construction d'une théorie: les représentations sociales. Revista Educação e Cultura Contemporânea, 12(27), 4-53.

HUDON, F. et GERVAIS, M. (2006). Réalités. Histoire et éducation à la citoyenneté (manuel de l'élève, 2B). Montréal, Québec: ERPI.

LAUTIER, N. (2006). L'histoire en situation didactique: une pluralité des registres de savoir. Dans V. Haas (dir.), Les savoirs du quotidien: transmission, appropriations, représentations (p. 75-88). Rennes, France: Presses universitaires de Rennes.

MANDELA, N. (2010). Long walk to freedom. The autobiography of Nelson Mandela. New York, NY: Back Bay Books.

MIHR, A. (2015). Why Holocaust education is not always human rights education. Journal of Human Rights, 14(4), 525-544.

MINISTÈRE DE L'ÉDUCATION, DU LOISIR ET DU SPORT (MELS). (2004). Histoire et éducation à la citoyenneté. Premier cycle. Québec: Gouvernement du Québec.

MINISTÈRE DE L'ÉDUCATION, DU LOISIR ET DU SPORT (MELS). (2010). Progression des apprentissages au secondaire. Histoire et éducation à la citoyenneté. $1^{\text {er }}$ cycle. Québec: Gouvernement du Québec. Repéré à http://www.education.gouv.qc.ca/ fileadmin/site_web/documents/education/jeunes/pfeq/PDA_PFEQ_histoire-educationcitoyennete-premier-cycle_2011.pdf

MOISAN, S. et SAUSSEZ, F. (2019). Pressions et expressions de normes dans les pratiques d'enseignement de l'histoire au secondaire. Recherches en éducation, 35, 92-109.

ORGANISATION DES NATIONS UNIES (2011). Déclaration des Nations Unies sur l'éducation et la formation aux droits de l'homme. Repéré à https://www. humanrights.ch/cms/upload/pdf/120110_texteFR_declarationUN_formationDH.pdf 
OSLER, A. et STARKEY, H. (2010). Teachers and human rights education. Oakhill, Royaume-Uni: Trentham Books.

ROCHER, G. (1992). Introduction à la sociologie générale. Montréal, Québec: Hurtubise.

SEIXAS, P. et MORTON, T. (2013). The big six historical thinking concepts. Toronto, Ontario: Nelson College Indigenous.

SWALWELL, K., PELLEGRINO, A. M. et VIEW, J. L. (2015). Teachers' curricular choices when teaching histories of oppressed people: Capturing the U.S. civil rights movement. The Journal of Social Studies Research, 39(2), 79-94.

TEEGER, C. (2015). "Both sides of the story": History education in post-apartheid South Africa. American Sociological Review, 80(6), 1175-1200.

TIBBITTS, F. (2002). Understanding what we do: Emerging models for human rights education. International Review of Education, 48(3), 159-171.

TIBBITTS, F. (2005). Transformative learning and human rights education: Taking a closer look. Intercultural Education, 16(2), 107-113.

VAN DER LEEUW-ROORD, J. (2014). Including a human rights focus into history teaching - The perspective of EUROCLIO. Dans C. Lenz, S. Brattland et L. Kvande (dir.), Crossing borders: Combining human rights education and history education (p. 67-78). Zurich, Suisse: Lit Verlag.

VESTERDAL, K. (2014). Human rights education through the lens of violation: Developing critical, active citizenship or constructing national identity? Dans C. Lenz, S. Brattland et L. Kvande (dir.), Crossing borders: Combining human rights education and history education (p. 151-174). Zurich, Suisse: Lit Verlag.

WANSINK, B., AKKERMAN, S., ZUIKER, I. etWUBBELS, T. (2018). Where does teaching multiperspectivity in history education begin and end? An analysis of the uses of temporality. Theory and Research in Social Education, 46(4), 495-527.

WASHINGTON, E. et HUMPHRIES, E. (2011). A social studies teacher's sense making of controversial issues discussions of race in a predominantly white, rural high school classroom. Theory and Research in Social Education, 39(1), 92-114.

WASSERMANN, J. (2017). The state and the volving of teaching about apartheid in school history in South Africa, Circa 1994-2016. Dans T. Epstein et C. Peck (dir.), Teaching and learning difficult histories in international contexts: A critical sociocultural approach (p. 59-71). Abingdon, Royaume-Uni: Taylor \& Francis Group. 
WOGENSTEIN, S. (2015). Holocaust education and human rights education reconsidered: A response to Anja Mihr. Journal of Human Rights, 14(4), 545-553.

ZEMBYLAS, M. (2015). 'Pedagogy of discomfort' and its ethical implications: The tensions of ethical violence in social justice education. Ethics and Education, 10(2), 163-174. 